\title{
The prevalence of elder abuse and associated factors among the elderly in Kashan city,
} Iran

\section{Zahra Khalili ${ }^{1}$, Mohsen Taghadosi ${ }^{2 *}$, Hamidreza Gilasi ${ }^{3}$, Ali Sadrollahi ${ }^{4}$}

1. Department of Nursing, Faculty of Medical Sciences, Ardabil University of Medical Sciences, Ardabil, Iran

2. Department of Nursing, Faculty of Medical Sciences, Kashan University of Medical Sciences, Kashan, Iran

3. Department of Epidemiology and Biostatistics, Kashan University of Medical Sciences, Kashan, Iran

4. Department of Clinical Affairs, Golestan University of Medical Sciences, Gorgan, Iran

*Corresponding author: Tel: +98 9133634439 Fax: +98 3615575058

Address: Department of Medical Surgical Nursing, Kashan University of Medical Sciences, Kashan, Iran E-mail: taghadosi_1345@yahoo.com

Received; 2014/11/28 revised; 2015/07/29 accepted; 2015/08/29

\begin{abstract}
Introduction: Elder abuse is among the major social health problems in today`s communities, and has significant effect on decreasing the health and security level in this group. The current study aimed to evaluate the prevalence and associated factors of abuse among the elderly in Kashan, Iran.

Materials and methods: This cross sectional study was conducted on 500 people over 60 years old in Kashan, Iran in 2014. Subjects were randomly selected from 10 healthcare centers of Kashan. Data were collected using elder abuse questionnaire. Cronbach alpha coefficient of the questionnaire was determined as 0.975. Data were analyzed by SPSS version 13. Pearson correlation coefficient and logistic regression analysis were used for data analysis. The statistically significant level was $\mathrm{P}<0.05$.

Results: Results of the current study showed that $80 \%$ of subjects have experienced at least one type of abuse during the last year. The highest rate of abuse was in the form of financial abuse $(45.6 \%)$, and the lowest was in ostracizing (16.6\%). There was a statistical significant relationship between the elder abuse and variables such as sex, age, number of children, marital status, living arrangement, residential situation, home properties, monthly income, insurance situation, illness history, walking ability and using mobility aids. Logistic regression analysis also showed significant relationship between the elder abuse and unemployment, living in an apartment, and the age range 71-80 years.

Conclusion: Considering the high prevalence of abuse among the elderly, clarification of this phenomenon is considered as one of the main priorities, which can be achieved only through multidisciplinary approach in the community, and needs cooperation and collaboration of all community members.
\end{abstract}

Keywords: Prevalence, Elder, Abuse

\section{Introduction}

Aging is one of the most serious problems in the field of healthcare (1). In recent years, social and economic changes, and scientific developments have increased life expectancy and decreased the rate of mortality, which caused significant increase in the elderly population (2). It is estimated that the elderly population of the world will be doubled within the next 40 years (3). This growth in the developing 
countries is far more than the developed countries. Countries it is estimated that the elderly population of Iran, as a developing country, will increase from $8.24 \%$ to $10 \%$, within 2011-2021(4). In addition to significant growth in the elderly population, no attention has been paid to their needs and problems as a vulnerable group of the society (5). Aging of population has many mental and emotional adverse consequences (6). Elder abuse is one of the most important and oldest problems of aging (7), which is considered as a widespread healthcare problem violating rights of the elderly (8). According to the world health organization (WHO), Elder abuse the inherent human rights and is a kind of intentional or unintentional violation in the forms of physical, mental, sexual, economical, discriminating, and neglecting(9). Problems caused by abuse are different in people with different races and cultures (10); unfortunately these problems are neglected by many authorities (7). Elder abuse not only causes physical damages, but it also has undesirable effects on the mental health of the aged (11), and threatens their health and safety (12). Studies reveal that abuse may cause mental disorders such as depression and anxiety, and decreases the life quality of the elderly $(8,13,14)$. Dong`s studies in Chicago showed that the elderly who encountered abuse had higher level of depression in comparison with the others $(13,15)$. According to the National Center on Elder Abuse of the USA, out of 10 American elderly, one experiences abuse, annually; while just one out of 14 is reported(16, 17). Prevalence of elder abuse is between $5 \%$ and $30 \%$, depending on the applied method and population under study $(8,18)$. The highest prevalence of elder abuse is reported regarding physical and extreme verbal violence in the USA and UK, respectively (19). A study in China showed that abuse among the Chinese elderly is $35 \%$ (13). In a review study, Cooper reported the prevalence of different types of elder abuse between $3.2 \%$ and $27.5 \%$ (20). The studies conducted in Iran also showed the elder abuse $(5,11,21-24)$. In this regard, Heravi reported $1.6 \%$ to $19.3 \%$ of abuse among the members Club of Tehran. He also reported that emotional and mental negligence and ostracizing and physical abuse had the highest and lowest prevalence rate, respectively (23-25). Karimi and Elahi also reported that the prevalence of physical violence and negligence of the elderly population of Ahwaz city were $10.5 \%$ and $31.7 \%$, respectively (21). Manouchehri et al. expressed that $84.4 \%$ of the elderly people referring to the parks of Tehran have experienced a type of emotional abuse (23). To evaluate the associated factors of elder abuse, Yuki Kishmoto stated that abuse among the Japanese elderly mostly associated with cognitive problems (26). Corina Naughton in Ireland reported that the most important abuse associated factors were unemployment and addiction of the care takers (27). While, Heravi believed that factors such as sex, age, education, insurance situation, employment and financial independence affect the level of abuse of the elderly (24). Another study showed that age is the main factor associated with the elder abuse; in a way that, with increase in age, there is an increase in the level of abuse (23). A study in China showed that the level of elder abuse had no association with the gender (28). But Naughton et al. reported that the level of elder abuse among women was higher than men (27). The study of Karimi, revealed that physical dependence was among the associated factors (21). $\mathrm{Li} \mathrm{Wu}$ et al. reported higher level of elder abuse among those who had lower education and a chronic disease, and lived alone (28). But Lachs, believed that living with family can be considered as a factor associated with abuse (8). Cultural differences influence elder abuse according to different reports (10). Age, sex, unemployment, insurance situation, education, level of depression, 
chronic disease and disabilities are among the factors associated with abuse $(2,15$, $23,27)$. Considering the impact of culture on elder abuse, diversity of the Iranian culture and national healthcare system, the important role of this subject on health promotion and improving quality of life in elderly and insufficient information in this issue motivated the researchers to design and conduct a study to determine the level of abuse and its associated factors among the elderly of Kashan city, Iran. It is hoped that the results of the current study help to identify the aspects of this problem and its associated factors.

\section{Materials and method}

Study population and sampling: In this cross-sectional study in 2014 the Prevalence of Elder abuse and Associated Factors among the Elderly in Kashan City were studied. The study population included 500 elderly aged over 60 years who had health care records in health centers in Kashan city. Kashan is a warm and dry city located in the edge of great desert in the center of Iran and city has about 200000 populations. According to previous study and estimation of inactivity in $32 \%$ of the elderly, confidence level of $95 \%(\mathrm{~d}=0.05, \mathrm{p}=0.32, \mathrm{z}=1.96)$ the sample size was calculated to be 334 according to following formula (18). It was increased to 1.5 fold due to cluster sampling, and finally 500 Individuals were investigated. Inclusion criteria were age over 60 years, Iranian nationality, ability to speak Persian, lack of mental disorder, having full awareness during the study, and ability to answer the questions. The elderly people who did not have the inclusion criteria were not included in the study (Equation 1).

Equation 1. $\quad \mathrm{n}=\left(\mathrm{z}_{1-\frac{\mathrm{a}}{2}}\right)^{2} \times \mathrm{p} \times \mathrm{q} / \mathrm{d} 2$

After coordination with the Department of Health and Medical Education and obtaining necessary permissions; the Kashan city was divided into 5 regions (center, north, south, west and east) based on map. From each region, two healthcare centers were randomly selected. In each health care centers the elderly were determined from family records and the subjects were selected randomly based on the population covered by the center. The selected subjects were evaluated by telephone calls. If they did not meet the inclusion criteria or were reluctant to participate in the study, another subject was replaced randomly. If the questionnaire had some missing data, the researchers contacted to the subject to complete the items and if it was not possible, another subject was replaced randomly. Then the researchers went to the houses of the subjects and after explaining the objectives of the study the questionnaire was completed. In elderly who did not have the ability to read and write the questionnaire was completed through interview.

Questionnaire: The first part of the questionnaire contained demographic data including variables (such as age, gender, number of children, educational level, marital status, living arrangements, occupational status and residential situation, Living arrangement, Economic dependency, chronic diseases, and use of mobility aids), were recorded. The second part of the questionnaire contained 49 items about in eight subscales such of negligence, psychological, physical, and financial abuse, deprivation of authorities, and ostracizing, and financial and emotional negligence.

This tool can be completed with the options as: yes, no, and never mind. Scores questionnaire are in the range of 0-100; the highest scores indicate the highest level of abuse. Therefore, score 100 shows the maximum level of abuse and zero points to the lack of evidence regarding abuse.

The lowest score as zero devoted to no answers and the highest score as number one devoted to yes. The option never mind had no score; therefore, this option was deleted in interviews. 
This tool was designed and validated based for the Iranian elderly who suffered from abuse, considering cultural characteristics of the Iranian community and found reliable, with satisfactory internal consistency (Cronbach $\alpha=0.97$ ) and testretest reliability $(r=0.99)(25)$.

Ethical Consideration: The study was approved by the Research Council and Research Ethics Committee of Kashan University of Medical Sciences, No. 92180 in 2014. The objectives of the study were explained to all the participants, and all of them signed a written informed consent before participation in the study. All the participants were informed that participation in the study is voluntary and were assured that their personal information would be treated confidentially. Researchers were committed to consider the participants rights in accordance to the principles explained in the Declaration of Helsinki.

\section{Statistical analysis}

The data was analyzed by SPSS version 13. The normality of the data set was analyzed by Kolmogorov-Smirnov test. Employing Pearson correlation coefficient; the logistic regression analysis was used for the multivariate analysis. The significance level for all the tests was considered $\mathrm{P}$ less than 0.05

\section{Results}

Out of 500 subjects participating in the current study, 290 (58\%) were men and $210(42 \%)$ were women. The mean age of the population under study was $72.07 \pm 9.03$ years, and the age ranged 60-70 years had the highest frequency (47.8\%). Considering education level, the highest frequency belonged to illiterate subjects $(61.2 \%)$ and only $38.8 \%$ were educated.
Most of the subjects $(71.8 \%)$ were unemployed and (44.2\%) lived independently. Most of them $(87.4 \%)$ were married, $76.8 \%$ had their own house. Of the total, $26.2 \%$ had no chronic diseases, $23 \%$ had diabetes, and $38 \%$ had high blood pressure; $3.6 \%$ and $4.6 \%$ reported history of stroke, and heart attack respectively, and $2.8 \%$ experienced bone fracture. Regarding mobility aids, $66 \%$ of the elderly used no mobility aids, $30.6 \%$ of them used reed and $3.4 \%$ used walker. The relationship between demographic and the associated factors, and the elder abuse is shown in Table 1. According to the results of Pearson test, there was a significant statistical relationship between the level of elder abuse and demographic variables such as age, gender, number of children, marital status, life conditions, living arrangements, home situation, monthly income, insurance situation, illness background, walking ability and using mobility aids. The prevalence of different types of elder abuse is shown in Table 2.

Among 500 participants, 400(80\%) reported that they had experienced at least one type of abuse in the past year. The highest rate of elder abuse was observed regarding financial abuse $(45.6 \%)$ and psychological abuse (45\%), and the lowest rate was measured for ostracizing (16.6\%) and physical abuse (22.2\%).

To control for confounding factors, logistic regression analysis was performed using elder abuse as the dependent variable, and all variable that reached a $\mathrm{P}$ value less than 0.05 in the Pearson test were examined as the independent variables. Result showed that elder abuse was significantly associated with unemployment, living in an apartment, age range 71-80 years (Table 3 ). 
Table 1. The relationship between demographic and the associated factors the elder abuse.

\begin{tabular}{|c|c|c|c|c|}
\hline Variable & Category & Confirmed elder abuse $^{\mathrm{a}}$ & No elder abuse $^{\mathrm{a}}$ & $\mathrm{P}$ value \\
\hline \multirow[t]{2}{*}{ Gender } & Female & $232(80)$ & $58(20)$ & 0.001 \\
\hline & Male & $168(80)$ & $42(20)$ & \\
\hline \multirow[t]{3}{*}{ Age (year) } & $60-70$ & $181(75.7)$ & $58(24.3)$ & 0.0001 \\
\hline & $71-80$ & $133(77.3)$ & $39(22.7)$ & \\
\hline & $>80$ & $86(96.6)$ & $3(3.4)$ & \\
\hline \multirow{3}{*}{$\begin{array}{l}\text { Number of } \\
\text { children }\end{array}$} & 0 & $11(68.8)$ & $5(31.2)$ & 0.001 \\
\hline & $1-4$ & $152(71.7)$ & $60(28.3)$ & \\
\hline & $>5$ & $237(87.1)$ & $35(12.9)$ & \\
\hline \multirow[t]{2}{*}{ Marital status } & Married & $342(78.3)$ & $95(21.7)$ & 0.01 \\
\hline & Widowed/single/separated & $58(92.1)$ & $5(7.9)$ & \\
\hline \multirow[t]{3}{*}{ Educational level } & Illiterate & $249(81.4)$ & $57(18.6)$ & 0.601 \\
\hline & Primary & $119(78.3)$ & $33(21.7)$ & \\
\hline & Diploma and above & $32(76.2)$ & $10(23.8)$ & \\
\hline \multirow{4}{*}{$\begin{array}{l}\text { Living } \\
\text { arrangement }\end{array}$} & Living with spouse & $169(74.4)$ & $58(25.6)$ & 0.001 \\
\hline & Living with spouse $\&$ children & $129(79.1)$ & $34(20.9)$ & \\
\hline & Living with children & $69(92)$ & $6(8)$ & \\
\hline & Living alone & $33(94.3)$ & $2(5.7)$ & \\
\hline \multirow[t]{3}{*}{ Job } & Unemployed & $295(82.2)$ & $64(17.8)$ & 0.151 \\
\hline & Employed & $54(75)$ & $18(25)$ & \\
\hline & Pensioner & $51(73.9)$ & $18(26.1)$ & \\
\hline \multirow{3}{*}{$\begin{array}{l}\text { Residential } \\
\text { situation }\end{array}$} & Leased & $42(75)$ & $14(25)$ & 0.001 \\
\hline & Landlord & 299(77.9) & $85(22.1)$ & \\
\hline & Children's home & $59(98.3)$ & $1(1.7)$ & \\
\hline \multirow[t]{3}{*}{ Home properties } & Apartment & $52(98.1)$ & $1(1.9)$ & $0.001^{\mathrm{b}}$ \\
\hline & Home without a yard & $103(81.7)$ & $23(18.3)$ & \\
\hline & Villa house & $245(76.3)$ & $76(23.7)$ & \\
\hline \multirow{3}{*}{$\begin{array}{l}\text { Economic } \\
\text { dependency }\end{array}$} & Independent & $170(76.9)$ & $51(23.1)$ & $0.225^{\mathrm{b}}$ \\
\hline & Dependent & $109(84.5)$ & $20(15.2)$ & \\
\hline & Support organizations & $121(80.7)$ & $29(19.3)$ & \\
\hline \multirow{2}{*}{$\begin{array}{l}\text { Having } \\
\text { insurance status }\end{array}$} & No & $129(92.8)$ & $10(7.2)$ & $0.001^{\mathrm{b}}$ \\
\hline & Yes & $271(75.1)$ & $90(24.9)$ & \\
\hline \multirow[t]{2}{*}{ Chronic disease } & No & $89(67.9)$ & $42(32.1)$ & $0.02^{\mathrm{b}}$ \\
\hline & Yes & $311(84.3)$ & $58(15.7)$ & \\
\hline \multirow[t]{2}{*}{ Walking ability } & Independent & $255(75.4)$ & $83(24.6)$ & $0.001^{\mathrm{b}}$ \\
\hline & Dependent & $145(89.5)$ & $17(10.5)$ & \\
\hline \multirow{2}{*}{$\begin{array}{l}\text { Use of mobility } \\
\text { aids }\end{array}$} & No & $225(77.3)$ & $75(22.7)$ & $0.004^{\mathrm{b}}$ \\
\hline & Yes & $145(85.3)$ & $25(14.7)$ & \\
\hline
\end{tabular}

${ }^{a}$ All data are presented as number or percent.

${ }^{\mathrm{b}}$ Pearson test.

Table 2. Prevalence of various types elder abuse.

\begin{tabular}{lcc}
\hline Any elder abuse & Confirmed elder abuse & No elder abuse \\
\hline Emotional abuse & $149(29.8)$ & $351(70.2)$ \\
Caregiver abuse & $178(35.6)$ & $322(64.4)$ \\
Financial neglect & $189(37.8)$ & $311(62.2)$ \\
Deprivation of authority & $206(41.2)$ & $294(58.8)$ \\
Psychological abuse & $225(45)$ & $275(55)$ \\
Financial abuse & $228(45.6)$ & $272(54.4)$ \\
Physical abuse & $111(22.2)$ & $389(77.8)$ \\
Ostracizing & $83(16.6)$ & $417(83.4)$ \\
Any abuse & $400(80)$ & $100(20)$ \\
\hline
\end{tabular}

All data are presented as number or percent. 
Table 3.The multivariate logistic regression analysis of odds ratios (OR) for elder abuse among elderly.

\begin{tabular}{lccccc}
\hline Characteristics & $\beta$ & SE & Wald x2 & P & OR (95\%CI) \\
\hline Age & & & & & \\
$71-80$ & -1.81 & 0.753 & 5.80 & 0.01 & $0.6(0.037-0.713)$ \\
\hline $\begin{array}{l}\text { Job } \\
\text { Unemployment }\end{array}$ & 0.976 & 0.479 & 4.15 & 0.04 & $5.75(1.03-6.78)$ \\
\hline $\begin{array}{l}\text { Home properties } \\
\text { Living in apartment }\end{array}$ & 2.31 & 1.11 & 4.33 & 0.03 & $88.75(1.14-89.90)$ \\
\hline
\end{tabular}

\section{Discussion}

In the current study, $80 \%$ of the subjects had experienced at least a type of abuse within the last year, a result which was quite different in comparison with the results obtained in the studies conducted in developed and even developing countries. This level of prevalence was higher than that of reported in Australia, Canada and England (3-10\%)(8), USA (4\% 10\%)(29), Korea $(6.3 \%)(30)$, Japan (17.9\%)(31), Hong Kong (23.5\%)(32), Russia (28.63\%)(33) and China (27.5\%)(34). Results of Manouchehri et al. showed that $87.8 \%$ of the population under study had experienced at least a type of abuse by their family members (6), which is higher than that of the current study. Abuse is an old issue associated with the elderly, but is rarely reported and has remained hidden due to lack of an appropriate definition and knowledge. Most of the conducted studies regarding mistreating the elderly have led to disagreement between the researchers and have created an obstacle to compare the results, due to employing different research methods, non-probability sampling, relative agreement on the concept of mistreating the elderly, using improper tools and difficulties associated with reliable data collection. It may be the reason for this statistical difference. The current study showed that financial and psychological abuses are the most common types of elder abuse, which is compatible with that of karimi et al.(21). Results of Manouchehri et al. showed that most of the subjects had experienced negligence and emotional abuse, and physical abuse was experienced the least (6). In the study by Zandi, financial abuse was the most common abuse among the elderly (35). On the other hand, in many other researches such as Oh (2), Rubio (36), and Amstadter (18) the psychological abuse was common. High prevalence of financial and psychological abuses among the Iranian elderly indicates the growth of personal and financial problems of the children in today`s modern life. Besides, economic crisis and inflation not only disable children to provide financial supports, but severe economic pressures may lead to financial abuse. The prevalence of financial abuse, $45.6 \%$, indicated the same result in the current study. Also, the present study showed that ostracizing and physical abuse had the lowest prevalence; the studies of Karimi(21), Manouchehri(6), Dong(37), Yan(34), and Rubio(36); confirmed this result. Similarities between the elder abuse in the developed countries and their differences with those of the developing countries probably indicate that elder abuse is affected by culture. It is noteworthy that since the other types of abuse are not usually visible, due to lack of knowledge they may not be considered as elder abuse, which results in more abuses. Elder abuse is a kind of severely complicated family violence, and many factors may affect it. In the current study, different associated variables, such as age range 70 to 80 years, having no children, living with the spouse, having home and adequate income, health insurance, hypertention, and physical disabilities were evaluated and the results showed significant statistical relationship between them and the level of elder abuse. Hence, results of the current study are compatible 
with those of the previous ones (24), and showed that the married elderly people and those who lived with their spouse encountered higher levels of abuse $(8,38)$. The study of $\mathrm{Li} \mathrm{Wu}$, showed that the elderly people who live alone usually experience higher levels of abuse (28), and other studies indicated that being widowed can be considered as the high risk for experiencing abuse (39); this may result from the fact that cohabitation increases the risk of conflicts and contacts; therefore, the level of abuse may increase in contrast. In the current study, elderly men were at higher risk of abuse than women; while, in the studies of $\mathrm{Li} \mathrm{Wu}$, Garre, and Nowrouzi women had experienced higher abuse $(28,40,41)$. This difference may result from different sampling methods, more men rather women in the population under study, or elderly women are more respectable and usually experience lower levels of abuse; since they do house hold responsibilities such as cooking or taking care of grandchildren. Also in the current study, adequate income, and having their own home were among the factors which increase the risk of financial abuse. $\mathrm{Li} \mathrm{Wu}$ reported that physical disability and chronic diseases are the factors for psychological abuse (28). According to this study, it was expected that elderly people who live alone or have a good financial situation experience lower level of abuse, but results showed that these people experience higher levels of abuse. This may result from high level of emotional abuse; these elderly people are usually neglected by their children since they are financially independent. Also, abusers may have financial problems and

\section{References}

1. Fotoukian Z, Mohammadi Shahboulaghi F, Fallahi Khoshknab M. Analytical on empowerment interventions in older people with chronic disease: A review be financially dependent on their elderly parents, which increase the level of abuse. Results of the current study showed no significant relationship between abuse, education level and job; while, in some other studies the level of education $(2,24)$ and having a job(24, 28), were considered as the factors associated with increasing abuse. Results of the current study showed the high prevalence of abuse among the elderly of Kashan, Iran, and at least eight out of ten elderly people experienced one type of abuse.

\section{Limitations}

The following cases are among the limitations of the current study: It was a cross-sectional study and to find the cause affect relationship, a prospective study should be conducted. Since the information regarding elder abuse was self-reporting it may result from perception and prejudice of the participants. In the current study the characteristics of abusers have not been evaluated, while mental disorder, using drugs and alcohol may increase the risk of abuse. Finally, the current study used an Iranian local tool and has considered even a type of abuse within the last year as elder abuse; therefore, it may have estimated elder abuse higher than real current situation.

\section{Acknowledgment}

This paper is derived from the M.Sc. thesis in Kashan University of Medical Sciences. The authors wish to thank the elderly who participated in the study for their cooperation.

literature. J Health Promot Manage. 2013;2(4):65-76.

2. Oh J, Kim HS, Martins D, Kim H. A study of elder abuse in Korea. Int J Nurs stud. 2006;43(2):203-14. 
3. WHO Centre for Health Development. Kobe J. Global health expectancy research among older people. 2001;1(2001): 10.

4. Farzianpour F, Foroushani AR, Badakhshan A, Gholipour M, Roknabadi EH. Quality of life for elderly residents in nursing homes. Glob J Health Sci. 2015;8(4):51676.

5. Adib-Hajbaghery M, Aghahoseini S. The evaluation of disability and its related factors among the elderly population in Kashan, Iran. BMC Public Health. 2007;7(1):261.

6. Rezai S, Manoochehri M. Comparison of mental disorders between home owner residents and nurse homes elders. Iran $\mathbf{J}$ Age. 2008;3(7):16-25.

7. Pérez-Rojo G, Izal M, Montorio I, Penhale B. Risk factors of elder abuse in a community dwelling Spanish sample. Arch Gerontol Geriatr. 2009;49(1):17-21.

8. Lachs MS, Pillemer K. Elder abuse. Lancet. 2004;364(9441): 1263-72.

9. Beach SR, Schulz R, Castle NG, Rosen J. Financial exploitation and psychological mistreatment among older adults: Differences between African Americans and Non-African Americans in a population-based survey. Gerontologist. 2010;50(6): 744-57.

10. Gharanjik A, Mohammadi F, Ansari G, Najafi F, Ghaderi S, Ashrafi K, et al . [The prevalence of depression in older Turkmen adults in 1389.] Salmand. 2011; 6 (3) :349. (Persian)

11. Heravi M, Anoosheh M, Frooghan M, Shaikhi MT, Hajizadeh E. Elder abuse by family members: An exploration in the lived experiences of older people. Iran $\mathbf{J}$ Nurs Res . 2010;5(18):6-17.

12. Dong X, Simon MA, Evans DA. Prevalence of self-neglect across gender, race, and socioeconomic status: findings from the Chicago health and aging project. Gerontology. 2011;58(3):258-68.

13. Dong $X$, Simon M, Beck T, Farran C, McCann J, Mendes de Leon C, et al. Elder abuse and mortality: The role of psychological and social wellbeing. Gerontology. 2010;57(6):549-58.

14. Dong X, Beck T, Simon MA. The associations of gender, depression and elder mistreatment in a communitydwelling Chinese population: the modifying effect of social support. Arch Gerontolc Geriatr. 2010;50(2):202-8.

15. Dong XQ, Simon M, Evans D. Crosssectional study of the characteristics of reported elder self-neglect in a community-dwelling population: findings from a population-based cohort. Gerontology. 2009;56(3):325-34.

16. Dong X, Simon MA. Association between elder abuse and use of ED: findings from the Chicago Health and Aging Project. Am J Emerg Med. 2013;31(4):693-8.

17. Clancy M, McDaid B, O'Neill D, O'Brien JG. National profiling of elder abuse referrals. Age Ageing. 2011;40(3):346-52.

18. Acierno R, Hernandez MA, Amstadter AB, Resnick HS, Steve K, Muzzy W, et al. Prevalence and correlates of emotional, physical, sexual, and financial abuse and potential neglect in the United States: the National Elder Mistreatment Study. Am J Public Health. 2010;100(2):292-7.

19. Comijs HC, Pot AM, Smit JH, Bouter LM, Jonker C. Elder abuse in the community: Prevalence and consequences. J Am Geriatr Soc. 1998;46(7):885-8.

20. Cooper C, Selwood A, Livingston G. The prevalence of elder abuse and neglect: a systematic review. Age Ageing. 2008;37(2):151-60.

21. Karimi M, Elahi N. Elderly abuse in Ahwaz city and its relationship with individual and social characteristics. Iran J Ageing. 2008;3(7):42-7.

22. Amir-Sadri A, Soleimani H. Elderly phenomena and its outcomes in IRAN. J Hygin Health. 2005;1(2):19-35.

23. Nikbakht Nasrabadi A, Hossein Abbasi N1, Mehrdad N. The prevalence of violence against Iranian women and its 
related factors. Glob J Health Sci. 2014;7(3):37-45.

24. Heravi Karimoei M. Frequency of elder abuse within family in members of Senior Social Clubs in Tehran City. Iran J Ageing. 2012;6(4):37-50.

25. Heravi-Karimooi M, Anoosheh M, Foroughan M, Sheykhi M, Hajizadeh E. [Designing and determining psychometric properties of the Domestic Elder Abuse Questionnaire.] Salmand. 2010; 5 (1) :721. (Persian)

26. Kishimoto $\mathrm{Y}$, Terada S, Takeda N, Oshima E, Honda H, Yoshida $\mathrm{H}$, et al. Abuse of people with cognitive impairment by family caregivers in Japan (a cross-sectional study). Psychiatry Res. 2013;209(3):699-704.

27. Naughton C, Drennan J, Lyons I, Lafferty A, Treacy M ,Phelan A, et al. Elder abuse and neglect in Ireland: results from a national prevalence survey. Age Ageing. 2012;41(1):98-103.

28. Wu L, Chen $\mathrm{H}, \mathrm{Hu} \mathrm{Y}$, Xiang $\mathrm{H}, \mathrm{Yu} \mathrm{X}$, Zhang T, et al. Prevalence and associated factors of elder mistreatment in a rural community in People's Republic of China: a cross-sectional study. PloS One. 2012;7(3):e33857.

29. Penhale B. Older women, domestic violence, and elder abuse: A review of commonalities, differences, and shared approaches. J Elder Abuse Negl. 2003;15(3-4):163-83.

30. Wang JJ. Psychological abuse and its characteristic correlates among elderly Taiwanese. Arch Gerontol Geriatr. 2006;42(3):307-18.

31. Anme T, McCall M, Tatara T. An exploratory study of abuse among frail elders using services in a small village in Japan. J Elder Abuse Negl. 2005;17(2):120.

32. Erlingsson CL, Carlson SL, Saveman BI. Perceptions of elder abuse: voices of professionals and volunteers in Swedenan exploratory study. Scand J Car Sci. 2006;20(2):151-9.

33. Puchkov P. Elder abuse: current research in the Russian Federation (2004-2006). J Adult Protect. 2006;8(4):4-12.

34. Yan EC-W, Tang CS-K. Elder abuse by caregivers: A study of prevalence and risk factors in Hong Kong Chinese families. J Fam Viol. 2004;19(5):269-77.

35. Staggs VS. Trends, victims, and injuries in injurious patient assaults on adult, geriatric, and child/adolescent psychiatric units in US hospitals, 2007-2013. Res Nurs Health. 2015;38(2):115-20.

36. Pérez-Cárceles M, Rubio L, Pereniguez J, Pérez-Flores D, Osuna E, Luna A. Suspicion of elder abuse in South Eastern Spain: The extent and risk factors. Arch Gerontol Geriatr. 2009;49(1):132-7.

37. Dong X, Simon MA, Gorbien M. Elder abuse and neglect in an urban Chinese population. J Elder Abuse Negl. 2007;19(3-4):79-96.

38. Paveza GJ, Cohen D, Eisdorfer C, Freels S, Semla T, Ashford JW, et al. Severe family violence and Alzheimer's disease: Prevalence and risk factors. Gerontologist. 1992;32(4):493-7.

39. Risco RC, Paniagua VMC, Jiménez MG, Poblador CM, Molina ML, Buitrago F. [Prevalence and risk factors of suspicion abuse in elder population]. Medicina Clinica. 2005;125(2):51-5.

40. Garre-Olmo J, Planas-Pujol X, López-Pousa S, Juvinyà $\mathrm{D}$, Vilà $\mathrm{A}$, Vilalta-Franch J. Prevalence and risk factors of suspected elder abuse subtypes in people aged 75 and older. J Am Geriatr Soc. 2009;57(5):815-22.

41. Oveisi S, Karimi R, Mahram M. Note from Iran: Self-reported elder abuse in Qazvin, 2012. J Elder Abuse Negl. 2014;26(3):337-40. 